\title{
Appréciation de la capacité de discernement des patients: procédure d'aide à l'usage des médecins
}

\author{
J.-B. Wasserfallen ${ }^{a}$, F. Stiefel $^{b}$, S. Clarke ${ }^{c}$, A. Crespo ${ }^{d}$
}

Tout acte médical est illicite sans le consentement libre et éclairé du patient, sauf s'il s'agit d'une urgence ou si le patient est incapable de discernement.

L'appréciation de la capacité de discernement, dans ses aspects intellectuels et volitifs, s'effectue par le médecin responsable de la prise en charge du patient, en fonction de la situation et de la question posée.

Si le médecin juge que le patient n'a pas son discernement, il documente le cas, recherche et traite une pathologie somatique ou psychiatrique associée à cette incapacité. Si le patient demeure sans capacité de discernement de manière durable, le médecin recherche s'il a déposé sa volonté par une des voies légales existantes. Si cette information manque, le médecin contacte la Justice de paix, pour obtenir un placement à fin d'assistance ou une mise sous tutelle/ curatelle, en rédigeant un certificat médical approprié.

Il est donc indispensable de connaître ces procédures.

\footnotetext{
a Direction médicale

b Service de Psychiatrie de liaison

c Division de Neuropsychologie

d Unité des Affaires Juridiques et Ethiques
}

Centre Hospitalier Universitaire Vaudois

Conflit d'intérêt: aucun

Correspondance:

Jean-Blaise Wasserfallen, MPP

CHUV

Direction médicale

Rue du Bugnon 46

CH-1011 Lausanne

tél. 0213141802

fax 0213141818

E-mail: jwasserf@chuv.hospvd.ch

\section{Introduction}

Tout acte médical (diagnostique et/ou thérapeutique) sur un patient est illicite sans son consentement libre et éclairé, sauf s'il s'agit d'une urgence ou si le patient est incapable de discernement [1].

L'application de ce principe est souvent à l'origine de problèmes pratiques, surtout avec le vieillissement de la population. Le Centre Hospitalier Universitaire Vaudois (CHUV) a voulu fournir une aide à la définition de la capacité de discernement, et à son évaluation chez les patients, pour faciliter les prises en charge cliniques dans les différents services. Ce travail a nécessité la mise en commun de connaissances médicales cliniques, psychiatriques, neuropsychologiques, et juridiques. Dans la mesure où cette problématique ne concerne pas uniquement les patients de notre établissement, sa diffusion pourrait être profitable à l'ensemble du corps médical, même si des variations liées aux différents systèmes juridiques cantonaux limitent sa validité générale.

\section{La capacité de discernement: définitions}

Le discernement est défini par l'article $16 \mathrm{du}$ Code Civil [2] comme suit:
«Toute personne qui n'est pas dépourvue de la faculté d'agir raisonnablement à cause de son jeune âge, ou qui n'en est pas privée par suite de maladie mentale, de faiblesse d'esprit, d'ivresse ou d'autres causes semblables, est capable de discernement dans le sens de la présente loi.»

Le discernement comprend deux aspects principaux [3]:

- Prise de conscience: le patient doit pouvoir comprendre l'information fournie, manipuler cette information rationnellement, et se rendre compte des conséquences de ses actes.

- Manifestation de sa volonté: le patient doit pouvoir communiquer ses choix et résister de manière adéquate à la pression exercée par autrui sur lui-même.

L'information doit être donnée dans un langage compréhensible, fournie sans pression, en laissant au patient un espace pour poser des questions, et en lui offrant un délai de réflexion. En effet, la manière de délivrer l'information conditionne en partie la réponse obtenue.

Ainsi, la capacité de discernement n'est pas forcément perturbée chez les patients atteints de troubles psychiques, hospitalisés en établissement psychiatrique, très âgés, hébergés en établissement médico-social, sous tutelle ou mineurs.

\section{Appréciation de la capacité de discernement}

La capacité de discernement doit s'apprécier de cas en cas par le médecin responsable de la prise en charge du patient. Elle est déterminée en fonction de la situation bien précise dans laquelle se trouve le patient et de la question qui se pose. Elle doit être évaluée chaque fois qu'une décision est prise.

En cas de doute sur la capacité de discernement, il s'agit d'évaluer en premier lieu l'état de conscience du patient, son orientation spatiotemporelle, personnelle et situationnelle. On 
Tableau 1

Le questionnaire de Silberfeld.

\begin{tabular}{|c|c|}
\hline Questions & Réponses \\
\hline 1. Pouvez-vous donner un résumé de la situation? & $\begin{array}{l}\text { Problème chronique (1) ou } \\
\text { Problème aigu (1) }\end{array}$ \\
\hline $\begin{array}{l}\text { 2. Quel traitement souhaiteriez-vous si vous vous trouviez } \\
\text { dans cette situation? }\end{array}$ & Réponse claire (1) \\
\hline 3. Pouvez-vous nommer un autre choix possible pour vous? & Un autre choix de traitement (1) \\
\hline 4. Quelles sont les raisons de votre choix? & Une raison valable (1) \\
\hline $\begin{array}{l}\text { 5. Quels sont les problèmes associés à votre choix } \\
\text { de traitement? }\end{array}$ & Un problème (1) \\
\hline 6. Que signifiera votre décision pour vous et votre famille? & $\begin{array}{l}\text { Pour le patient (1) } \\
\text { Pour la famille (1) }\end{array}$ \\
\hline 7. Quel effet à court terme aura le traitement? & Effet à court terme (1) \\
\hline 8. Pouvez-vous penser à un effet à long terme? & Effet à long terme (1) \\
\hline 9. Pouvez-vous répéter quel traitement vous souhaitez? & $\begin{array}{l}\text { Répétition de la réponse } \\
\text { à la question } 2 \text { (1) }\end{array}$ \\
\hline
\end{tabular}

doit aussi s'assurer de la compréhension générale que le patient a de sa maladie, de sa capacité d'apprécier les conséquences probables d'une décision, et de gérer l'information de manière rationnelle. Il doit avoir la faculté de maintenir et de communiquer ses choix.

Dans les situations où cette appréciation s'avère difficile, le questionnaire de Silberfeld [4] peut être utilisé comme guide (tab. 1). Un score $\geq 6 / 10$ indique que le patient peut être considéré comme compétent.

Il faut être conscient qu'un refus inadéquat de traitement n'est pas forcément dû à une incapacité de discernement, mais peut être l'expression d'un conflit entre le patient et l'équipe ou bien refléter un état anxieux, d'irritation ou de révolte. L'évaluation de la capacité du discernement doit donc tenir compte de ces éléments.

Si le médecin juge que le patient n'a pas son discernement, il est important de documenter le cas et de décrire précisément dans le dossier quels éléments ont appuyé ce jugement.

\section{Procédure de bilan de l'incapacité de discernement}

Cette procédure peut nécessiter quatre étapes.

\section{Documentation des éléments évocateurs de l'incapacité de discernement}

Symptômes, signes, autres éléments et score de Silberfeld sont à noter dans le dossier médical.

\section{Exclusion d'une lésion cérébrale}

Si une lésion cérébrale est vraisemblable, elle peut entraîner des troubles de compréhension, de mémoire ou praxiques secondaires, qui doivent être documentés par un bilan neuropsychologique, dans le but d'apprécier leur importance et l'impact qu'ils peuvent avoir sur la capacité de discernement. Si le bilan neuropsychologique révèle des troubles cognitifs ou praxiques, une imagerie cérébrale par CT-scan ou IRM est indiquée pour s'assurer de l'absence d'une lésion organique potentiellement traitable.

\section{Exclusion d'un état confusionnel médicamenteux et/ou carentiel}

Si une étiologie médicamenteuse ou carentielle est vraisemblable, le facteur causal doit être tout d'abord corrigé, et une réévaluation clinique agendée pour effectuer un nouveau bilan. Là encore, un bilan neuropsychologique peut parfois s'avérer utile comme aide à la décision.

\section{Exclusion d'une autre affection psychiatrique}

Une fois les causes somatiques écartées, une affection psychiatrique doit être envisagée et recherchée, dans la mesure où elle pourrait expliquer l'incapacité de discernement du patient.

\section{Quand faut-il appeler le psychiatre?}

Lorsque le médecin responsable de la prise en charge n'est pas absolument satisfait de son évaluation, le recours à un psychiatre peut être utile, car il apporte non seulement un regard d'expert mais également un regard neutre et un espace supplémentaire qui permet de réévaluer la situation avec le patient et l'équipe.

En cas d'affection psychiatrique importante, et dans la mesure où l'équipe hospitalière et le médecin responsable de la prise en charge n'ont pas l'habitude d'une telle situation, nous leur conseillons de faire appel au Service de psychiatrie de liaison autant pour aider à évaluer la capacité de discernement que pour assurer une prise en charge adéquate. Rappelons à ce stade que le diagnostic psychiatrique est un élément prépondérant dans l'évaluation de la capacité de discernement, mais qu'il ne constitue pas en soi une raison suffisante pour justifier une incapacité. Seule l'évaluation de la capacité de discernement compte.

Que faire si le patient refuse de voir un psychiatre? Dans les cas où il y a matière à confusion ou à contentieux et que le patient refuse de voir un psychiatre, ce dernier peut être inclus dans une réunion pluridisciplinaire pour soutenir le médecin traitant et l'équipe dans leur approche 
du patient, leur procédure d'appréciation et la prise de décisions.

Dans le cas où un patient refuse tout traitement ou menace même de quitter l'hôpital contre avis médical, il peut s'agir d'un état anxieux, d'une émotion forte qui se manifeste par un passage à l'acte, ou d'un conflit entre le patient et le médecin et/ou l'équipe infirmière. Il est souvent possible de désamorcer la situation en laissant le patient exprimer ses émotions et ses attentes envers la médecine et les soignants, avec ou sans l'aide du psychiatre.

S'il y a suspicion d'une psychopathologie importante (trouble psychotique, par exemple) et d'un comportement qui met le patient ou d'autres personnes en danger, une consultation psychiatrique, voire des mesures de contrainte peuvent être nécessaires, même contre la volonté du patient.

\section{Procédure à suivre une fois l'incapacité de discernement documentée}

Une fois l'incapacité de discernement documentée, il s'agit de rechercher si le patient a manifesté sa volonté de manière anticipée. Il a pour ce faire diverses possibilités: des directives anticipées écrites, qu'il devrait avoir laissé à ses proches et/ou à son médecin traitant [5]; un représentant thérapeutique au sens de la nouvelle Loi sur la Santé Publique du Canton de Vaud [5], désigné par écrit; un représentant légal si une mesure de privation de liberté s'est déjà imposée (curatelle, tutelle, placement à fin d'assistance); finalement, les informations que pourraient fournir les proches sur les préférences du patient peuvent être sollicitées et recueillies, tout en sachant que leur avis personnel n'a pas de caractère contraignant ni obligatoire.

Il faut toutefois se souvenir qu'en cas d'urgence, le médecin est tenu d'agir au mieux des intérêts objectifs du patient en tenant compte de sa volonté présumée [6].

Si ces documents n'existent pas ou si ces démarches n'aboutissent pas, et ne permettent pas de résoudre le problème posé, la situation peut devenir délicate dans la mesure où les procédures diagnostiques ou thérapeutiques envisagées restent illicites dans l'esprit de la loi. D'autres mesures s'imposent si cet état de fait persiste, et le patient doit être protégé par des mesures légales comme la mise sous curatelle ou tutelle.

Le cas particulier du maintien à domicile d'un patient sans capacité de discernement doit également être mentionné ici, dans la mesure où le médecin traitant peut être appelé à intervenir pour évaluer ce genre de situation, et prendre des mesures pour protéger le patient en demandant un placement à fin d'assistance.

Dans ces deux cas de figure, le médecin peut contacter l'assistante sociale, qui servira de relais avec la Justice de paix de la commune de domicile du patient pour obtenir ou un placement à fin d'assistance ou une mise sous tutelle/curatelle.

Le médecin transmet à l'assistante sociale l'original du certificat médical établissant l'incapacité de discernement, daté et signé. En vue d'un placement à fin d'assistance, le médecin doit décrire les troubles mentaux et/ou les symptômes que présente le patient, attester que sa capacité de discernement est de ce fait altérée, l'empêchant de gérer ses intérêts et de désigner valablement une personne pour la représenter. Il doit par ailleurs attester que son état constitue un danger pour lui-même ou pour autrui, de manière détaillée et conclure qu'il n'est actuellement pas en mesure d'être entendu par la Justice de paix. Pour une mise sous tutelle ou curatelle, le médecin doit fournir un certificat médical similaire, à la différence près que l'état du patient ne constituant pas un danger pour luimême ou autrui, ce paragraphe ne figure pas au certificat médical.

\section{Discussion}

La capacité de discernement du patient est un élément essentiel dans sa prise en charge. En effet, en son absence, et sauf en cas d'urgence, tout acte diagnostique et/ou thérapeutique est illicite [1]. La capacité de discernement est une notion difficile à définir, qui varie en fonction du type de décision à prendre, et doit donc être l'objet d'évaluations itératives. Toutefois, celles-ci prennent un temps important, peu compatible avec l'évolution de la pratique médicale, dans laquelle des contraintes de temps sont de plus en plus présentes et imposées par le système de santé. Il nous semblait important de pouvoir mettre à disposition des cliniciens un document d'aide à l'évaluation, qui soit simple, sensible, spécifique, et donc facile à utiliser.

La littérature est relativement pauvre sur le sujet et centrée sur deux aspects: le consentement du patient au traitement [7] et la rédaction de directives anticipées $[3,4]$. Même si ces deux sujets sont relativement différents, ils présentent une importante similitude, la capacité du patient de se positionner face à une situation dans laquelle une décision peut avoir des conséquences 
importantes et même engager son avenir. C'est pourquoi nous estimons que le questionnaire de Silberfeld proposé [4], même s'il a été développé pour l'établissement de directives anticipées, est applicable à l'obtention du consentement du patient à une procédure diagnostique et thérapeutique.

La procédure à suivre avec la Justice de paix peut présenter certaines différences suivant les cantons, puisqu'elle est réglée par le droit cantonal. Nous n'avons pas exploré ce domaine, nous contentant de décrire ici celle qui est applicable dans le canton de Vaud et laissant au lecteur le soin de l'adapter à la situation qui prévaut dans son canton.

Il nous semble essentiel que cette notion soit mieux intégrée dans la pratique médicale pour éviter les conséquences pénibles, à la fois sur le plan humain et sur le plan médico-légal, que pourrait entraîner la réalisation d'une procédure diagnostique et/ou thérapeutique sans le consentement éclairé du patient, en dehors d'une situation d'urgence. Si le projet de révision du Code civil concernant le traitement des patients incapables de discernement et la nouvelle conception de la privation de liberté à des fins d'assistance est adopté [8], cette procédure d'évaluation prendra encore plus d'importance dans l'activité clinique quotidienne.

\section{Références}

1 Loi sur la Santé Publique du canton de Vaud, article 23 .

2 Code Civil Suisse, article 16.

3 Fazel S, Hope T, Jacoby R. Assessment of competence to complete advance directives: validation of a patient centred approach. Br Med J 1999; 318:493-7.

4 Silberfeld M, Nash C, Singer PA. Capacity to complete an advance directive. J Am Geriatr Soc 1993;41:1141-3.

5 Loi sur la Santé Publique du canton de Vaud, article $23 \mathrm{a}, \mathrm{b}, \mathrm{c}$.

6 Code Civil Suisse, article 397, lettres A à F

7 Appelbaum PS, Grisso T. Assessing patient's capacities to consent to treatment. N Engl J Med 1988;319:1635-8.

8 Steiner U, Kuhn H. Révision du Code civil. Traitement des patients incapables de discernement et nouvelle conception de la privation de liberté à des fins d'assistance. Bull Méd Suisses 2003;84(48):2543-8. 